\title{
Detection of non-ST-elevation myocardial infarction and unstable angina in the acute setting: meta-analysis of diagnostic performance of multi-detector computed tomographic angiography
} Piet K Vanhoenacker*†1, Isabel Decramer ${ }^{\dagger 1,2}$, Olivier Bladt ${ }^{1}$, Giovanna Sarno ${ }^{2}$, Charlotte Bevernage ${ }^{1}$ and William Wijns ${ }^{2}$

Address: ${ }^{1}$ Department of Radiology and Medical Imaging, Onze Lieve Vrouw Ziekenhuis, Aalst, Belgium and ${ }^{2}$ Cardiovascular Center Aalst, Aalst, Belgium

Email: Piet K Vanhoenacker* - piet@vanhoenacker.be; Isabel Decramer - i_decramer@yahoo.com; Olivier Bladt - olibladt@hotmail.com; Giovanna Sarno - giovannasarno@yahoo.it; Charlotte Bevernage - charlotte_bevernage@yahoo.com; William Wijns -William.Wijns@olvzaalst.be

* Corresponding author †Equal contributors

Published: 19 December 2007

BMC Cardiovascular Disorders 2007, 7:39 doi:10.1/86/147|-2261-7-39

This article is available from: http://www.biomedcentral.com/147I-226I/7/39

(c) 2007 Vanhoenacker et al; licensee BioMed Central Ltd.

This is an Open Access article distributed under the terms of the Creative Commons Attribution License (http://creativecommons.org/licenses/by/2.0), which permits unrestricted use, distribution, and reproduction in any medium, provided the original work is properly cited.
Received: 30 August 2007

Accepted: 19 December 2007

\begin{abstract}
Background: Multi-detector computed tomography angiography (MDCTA) has been increasingly used in the evaluation of the coronary arteries. The purpose of this study was to review the literature on the diagnostic performance of MDCTA in the acute setting, for the detection of non-ST-elevation myocardial infarction (NSTEMI) and unstable angina pectoris (UAP).

Methods: A Pubmed and manual search of the literature published between January 2000 and June 2007 was performed. Studies were included that compared MDCTA with clinical outcome and/or CA in patients with acute chest pain, presenting at the emergency department. More specifically, studies that only included patients with initially negative cardiac enzymes suspected of having NSTEMI or UAP were included. Summary estimates of diagnostic odds ratio (DOR), sensitivity and specificity, negative (NLR) and positive likelihood ratio (PLR) were calculated on a patient basis. Random-effects models and summary receiver operating curve (SROC) analysis were used to assess the diagnostic performance of MDCTA with 4 detectors or more. The proportion of non assessable scans (NAP) on MDCTA was also evaluated. In addition, the influence of study characteristics of each study on diagnostic performance and NAP was investigated with multivariable logistic regression.

Results: Nine studies totalling 566 patients, were included in the meta-analysis: one randomised trial and eight prospective cohort studies. Five studies on 64-detector MDCTA and 4 studies on MDCTA with less than 64 detectors were included ( 32 detectors $n=1,16$ detectors $n=2,16$ and 4 detectors $n=1$ ). Pooled DOR was $131.8 \mathrm{I}(95 \% \mathrm{Cl}, 50.90-34 \mid .3 \mathrm{I})$. The pooled sensitivity and specificity were $0.95(95 \% \mathrm{Cl}, 0.90-0.98)$ and 0.90 $(95 \% \mathrm{Cl}, 0.87-0.93)$. The pooled NLR and PLR were $0.12(95 \% \mathrm{Cl}, 0.06-0.2 \mathrm{I})$ and $8,60(95 \% \mathrm{Cl}, 5.03-14,69)$.

The results of the logistic regressions showed that none of the investigated variables had influence on the diagnostic performance or NAP

Conclusion: MDCTA of the coronary arteries performs good to excellent in the diagnosis of coronary artery disease in the acute setting and it can be used for early exclusion of NSTEMI or UAP in patients in the emergency department.
\end{abstract}




\section{Background}

Acute chest pain accounts for approximately $6.5 \%$ of all emergency department visits in the US $[1,2]$. Failure to diagnose myocardial ischemia as a cause of acute chest pain has serious implications and the triage of patients with possible ischemia is often difficult. To reduce diagnostic error, many patients that present at the emergency department are admitted for observation, even when no initial ECG changes or elevated cardiac enzymes are present. Emergency departments have therefore developed chest pain units and diagnostic protocols commonly including serial cardiac enzyme evaluations and ECG's, supplemented with some form of stress testing with or without imaging [3]. Many of these patients are found to have no acute coronary syndrome (ACS) and more than 2 million patients with acute chest pain are admitted to the hospital without developing an ACS $[4,5]$. Data from Germany reveal that the number of potentially unnecessary hospital days is high, amounting to as much as 839 per 100 patients admitted for acute chest pain [6].

Non invasive access to coronary anatomy has become available with the emergence of multi-detector computed tomography (MDCTA) of the coronary arteries. Diagnostic performance of MDCTA has been evaluated in many studies [7]. Even though appropriate indications for MDCTA remain largely work in progress, the technique has been used as a tool to rule out ACS in the emergency department. [8-16]

The purpose of our study was to review the literature and perform a meta-analysis on the diagnostic performance of multi-detector computed tomography angiography for the exclusion of ACS in the emergency department. More specifically, we focused on the early non-invasive diagnosis of non ST elevation myocardial infarction (NSTEMI) with initially negative biomarkers and unstable angina pectoris (UAP). A second aim was to investigate the influence of multiple independent study-related variables on the diagnostic performance of MDCTA.

\section{Methods \\ Study selection}

A search in the Computer Retrieval of Information on Scientific Projects (CRISP), Database of Abstracts of Reviews of Effects (DARE), International Network of Agencies for Health Technology Assessment (INAHTA) and Cochrane Database of Systematic Reviews (CDSR) databases was done from January 1998 to June 2007. The purpose was to reveal the existence of structured reviews on MDCTA for ruling out ACS in the emergency department. A structured review or systematic review is based on a thorough review of the literature concerning a single topic but differs from a narrative review by statistically combining the results of several studies into a single outcome measure, by using techniques of meta-analysis. To search for original articles, a structured search of the PUBMED database from January 1998 to April 2007 was performed using an elaborated form of a previously described search strategy [17], by two authors (PV, OB). A second search was done by the same authors with another method recently described [18]. Both strategies can be found in Additional file 1 . Reference lists of review articles and cited articles were used to locate additional studies. The following journals were hand-searched from January 1998 to March 2007: European Radiology, Radiology, Radiographics, American Journal of Roentgenology, Journal of Computer Assisted Tomography, Journal de Radiologie, Heart, The Lancet, New England Journal of medicine, JAMA, Journal of the American College of Cardiology, American Journal of Cardiology, American Heart Journal, Circulation, Hypertension, Circulation Research, European Heart Journal, British Medical Journal, Journal of Nuclear Cardiology, Emergency Medicine English Dutch, French, German, Italian and Spanish articles were also potentially included because the authors were familiar with these languages. Finally the results of all the searches were fused with bibliography software and duplicate publications were automatically removed (Reference Manager Professional edition version 10, ISI research software).

Studies were included in the meta-analysis if they met the following inclusion criteria: the data were acquired with a multi-detector CT-scanner with at least four detectors; catheter angiography was used as the reference standard and/or clinical follow up was obtained in all patients concerning the presence of UAP or NSTEMI ; the criteria for a positive result of MDCTA and CA were explicitly defined as 50 percent or greater diameter stenosis; the criteria for a positive clinical outcome (diagnosis of NSTEMI or UAP) where coherent with actual clinical standards and were explicitly defined; the absolute numbers of true-positive, false-negative, false-positive, and true-negative test results were available or could be derived from the available data or from the authors. These absolute numbers were accepted if they were derived on a per segment basis, or on a per patient basis. For segmental analysis an adapted American Heart Association 15 segment scheme of the coronary tree was used [19].

Exclusion criteria were: not an acute ACS setting or MDCTA later than 24 hours after the onset of the acute event; unknown status of cardiac biomarkers or positive markers; review article; not all patients were tested with the reference test (Table 1).

The results of this search were analysed by two independent radiologists $(\mathrm{PV}, \mathrm{OB})$ as follows: Each investigator independently evaluated the retrieved studies for possible inclusion. In the case of conflicting findings as to whether 
Table I: Number and reasons for exclusion of articles that were reviewed in full-text.

$\begin{array}{lr}\text { Not an acute ACS setting } & 71 \\ \text { Unknown status of/or positive biomarkers } & 6 \\ \text { Review article } & 6 \\ \text { No comparison with reference standard in all cases } & \text { I } \\ \text { Total } & 84\end{array}$

a paper should be included, a decision was reached by consensus. In a first round articles were eliminated that clearly did not match the inclusion criteria, on the basis of the title or the abstract. In a second round, hard copies of the papers that gave rise to doubt on the basis of their abstracts were obtained and the full text was read, again eliminating a group of papers. The final group consisted of the included papers.

Inclusion of studies was guided by the quality of the study design and report. A formal system for quality evaluation, Quality Assessment of Diagnostic Accuracy Studies (QUADAS, 20), with a maximum of 14 points was used for judging quality in the final evaluation of included papers (Fig. 1 , round 3 ). A score of $>/=12$ was considered acceptable.

\section{Data extraction}

The study parameters where extracted first independently and subsequently by consensus if a disagreement existed between the observers concerning the numeric value of a parameter (PV, ID). Data were extracted from the original articles taking into account the Standards for Reporting of Diagnostic Accuracy (STARD) checklist [21].

The absolute numbers of FN, FP, TP, TN were retrieved, calculated or requested from the authors. The numbers were calculated with Bayes theorem if only values for sensitivity, specificity, and predictive values were reported. This was done separately for per patient and per segment data, if available.

MDCTA was considered true-positive per patient, or per segment if at least one significant stenosis ( $>=50 \%$ diameter stenosis) was found on MDCTA in, respectively, the investigated patient, or segment and confirmed on CA. MDCTA was considered true-negative if significant stenoses were correctly ruled out. MDCTA was considered false-negative if no significant stenoses were found on MDCTA and CA revealed at least one significant stenosis. MDCTA was considered false-positive if it revealed at least one stenotic stenosis and CA showed no significant stenoses. If no CA was done, positive clinical outcome of UAP or NSTEMI was the positive reference identifier, and absence of UAP or NSTEMI was the negative reference identifier with an analogue classification scheme as above.

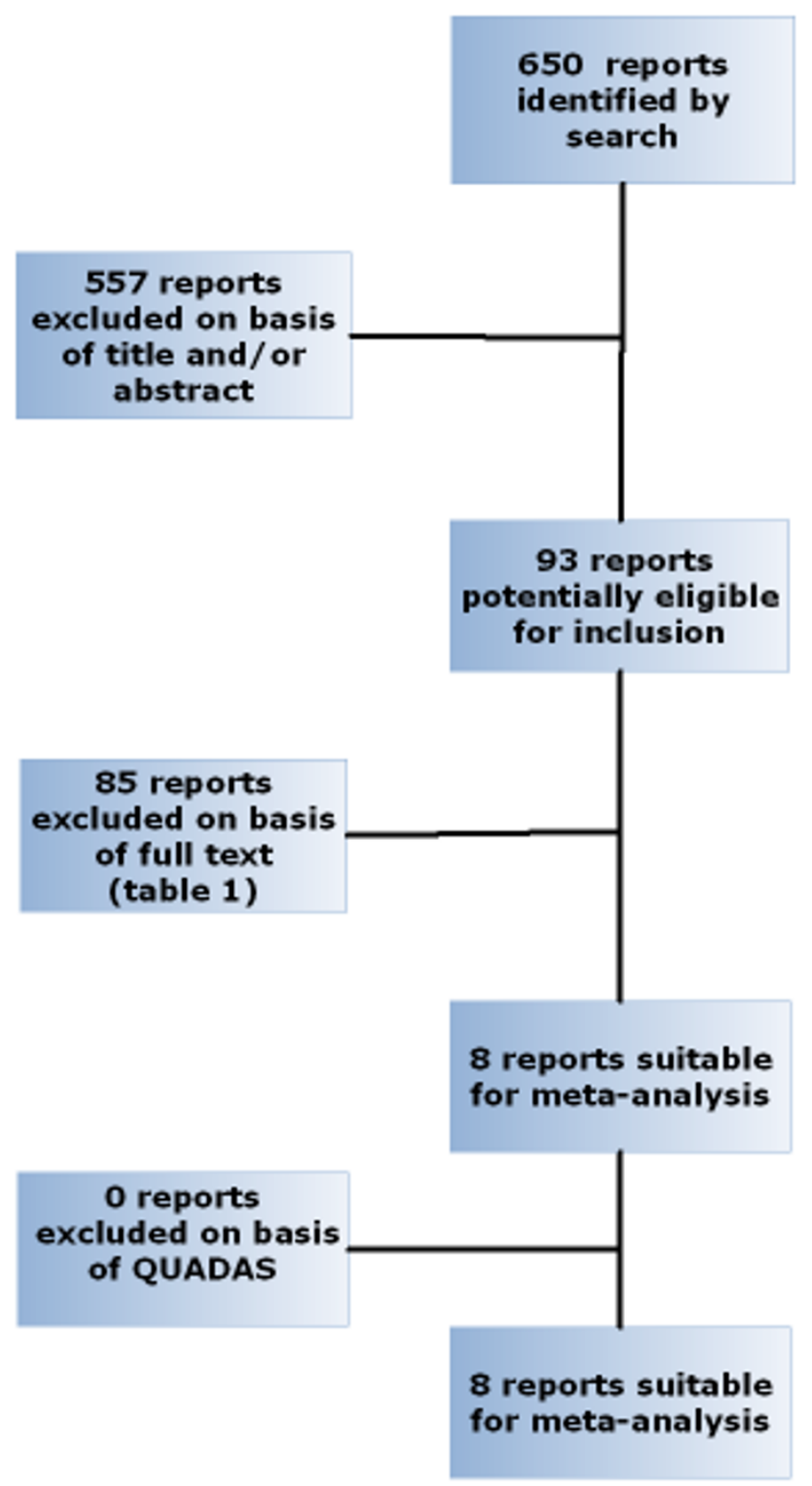

Figure I

Flow diagram of the reviewing process.

Additional study characteristics were extracted (Table 2).

\section{Data synthesis and statistical analysis}

Results are expressed as mean with 95\% confidence intervals $(95 \% \mathrm{CI})$, unless otherwise specified.

Interobserver agreement for study selection was evaluated with Cohen's Kappa test in which a value higher than 0.8 is considered to imply very good to excellent agreement. The three rounds of selection were evaluated. 
Table 2: Study Characteristics

\begin{tabular}{|c|c|c|c|c|c|c|c|c|c|c|}
\hline & Year & Type & Det & Age & $M / F$ & Prob & INC & NAP & Outc & $\mathrm{NCA}$ \\
\hline White $^{8}$ & 2005 & $\mathrm{PC}$ & 16 & 51 & 1.03 & All** & 69 & 0 & CLIN & - \\
\hline Gallagher ${ }^{9}$ & 2006 & $\mathrm{PC}$ & 64 & 49 & 0.53 & Low & 96 & 0.07 & CLIN & 5 \\
\hline Hoffmann 10 & 2006 & $\mathrm{PC}$ & $16 / 64$ & 57 & 0.53 & All** & 40 & 0 & CLIN & - \\
\hline Hoffmann"I & 2006 & $\mathrm{PC}$ & 64 & 54 & 0.58 & All** & 106 & 0.03 & CLIN & 8 \\
\hline Olivetti 12 & 2006 & $\mathrm{PC}$ & 16 & 59 & 0.61 & $U$ & 31 & 0 & CA & 31 \\
\hline Sato ${ }^{13}$ & 2006 & $\mathrm{PC}$ & $4 / 16$ & 60 & 0.85 & $\cup$ & 34 & 0.09 & CLIN & - \\
\hline Goldstein 14 & 2007 & $\mathrm{RCT}$ & 64 & 48 & 0.43 & Low & 99 & 0.11 & CLIN & 12 \\
\hline Meijboom ${ }^{15}$ & 2007 & $\mathrm{PC}$ & 64 & 59 & 0,76 & Low & 33 & 0 & $\mathrm{CA}$ & $33^{*}$ \\
\hline Rubinshtein 16 & 2007 & $\mathrm{PC}$ & 64 & 56 & 1.76 & Int & 58 & 0 & CLIN & 17 \\
\hline
\end{tabular}

Type: Study type.

RCT: Randomized controlled trial.

PC: Prospective cohort.

Det: Number of detectors.

M/F: Male/Female ratio.

Prob: Pre-test probability.

INC: Number of included patients.

NAP: Non assessable proportion of patients = Ratio of non-diagnostic versus included patients.

Outc: reference standard used.

NCA: Number of patients that underwent Conventional Angiography.

Clin: Clinical.

U: Unknown.

*:only a fraction of the patients were included: the patients that clearly had negative biomarkers and were categorized as "low risk" in this study.

**: included patients with pre-test probability ranging from low to high

The main analysis was performed at the patient level, as most studies focused on this level of information. We evaluated potential heterogeneity and inconsistency between publications $[22,23]$ expressed with the Higgins and Thompson index which calculates the $\mathrm{I}^{2}$ statistic, and is a derivative of Cochran's Q [24-26]. Cochran's Q displays a low power for detection of inconsistency when the number of studies is low, and a too high power when the number of studies is high.

Publication bias was assessed according to the method introduced by Sterne and Egger $[27,28]$. The existence of publication bias is expressed as an intercept value, and is 0 if no publication bias is found. A Funnel plot for graphic analysis of publication bias was constructed. A funnel plot is a plot of some measure of each study's sample size, such as the standard error, as function of its effect size. A distribution of the datapoints as an inverted funnel indicates that publication bias is highly unlikely. Calculations were performed with Statsdirect (StatsDirect Ltd. StatsDirect statistical software, England).

Summary estimates for sensitivity, specificity, positive likelihood ratio (PLR), negative likelihood ratio (NLR) and the overall diagnostic performance expressed in the diagnostic odds ratio (DOR) were calculated. This was done with a random effects model, which takes into account the variability between studies [29].
Subsequently, we performed a random effects SROC analysis to estimate the relationship between sensitivity and specificity, taking into account potential differences in positivity criterion (that is, the threshold used to mark a test as positive) and other factors of heterogeneity between settings. In a SROC analysis the logits (log odds) of sensitivity and 1-specificity are summed to calculate D, the log of the diagnostic odds ratio, and the logits are subtracted to calculate $S$, a proxy for the positivity criterion of the diagnostic test [30-32]. Then, a linear regression model $\mathrm{D}=\mathrm{a}+\mathrm{bS}$ is estimated, weighted by the inverse of the variance of $D$. Values for area under the curve (AUC) and $\mathrm{Q}^{*}$ were calculated with their respective standard error (SE). $\mathrm{Q}^{*}$ is the point of intersection of the SROC curve where SE and SP are equal and is a statistic that describes global diagnostic performance [33].

A multivariable logistic meta-regression was performed to investigate the influence of multiple explanatory variables on the diagnostic performance.

The evaluated variables included: Male/female ratio, mean age of patients, number of included patients, outcome used (CA or clinical), non-assessable patients excluded before analysis of diagnostic performance coded as a dichotomous variable (yes/no), number of detectors in the scanner used and pre-test probability of the cohort (low, intermediate, high, all or unknown) as indicated by the authors. Other variables such as scan and technique related variables were not investigated since in a prior 
meta-analysis these variables did not show any important influence [34] or could not be obtained in detail (such as coronary calcium score or pre-test probability). Variables with a significance level of $\mathrm{p}<=0.10$ were added to the multivariable meta-regression model in a stepwise forward manner. A variable was kept in the model if $\mathrm{p}<0.05$. A p $<=0.10$ was used to add variables to the multivariable model whereas a $\mathrm{p}<=0.05$ was used to retain variables in the model. For adding variables to the model a higher pvalue was chosen so as to increase the power of finding important effects. The beta-coefficients and corresponding relative diagnostic odds ratios from the meta-regression analysis indicated the effect of each variable on the overall diagnostic performance.

The proportion of non-assessable patients (NAP) was pooled with a random effects model and evaluated as dependent variable in a multivariable regression analysis to evaluate which variables influenced this proportion. NAP was defined as the ratio of technical failure or nondiagnostic scans to the final number of included patients (Fig 2). The same variables except for the exclusion of non-assessable segments were tested as above. Software used for the calculation of pooled estimates, SROC analyses and all regressions was Meta-DiSc (version 1.3 Clinical Biostatistics Unit-Hospital Ramon y Cajal, Madrid, Spain) and/or Statsdirect

\section{Results}

\section{Study selection and data extraction}

The search in the CRISP, DARE, INAHTA and CDSR databases revealed no structured reviews or meta-analyses on the diagnostic performance of MDCTA for the assessment of NSTEMI or UAP in the acute setting.

The Pub-med search and the manual search for original articles resulted in 650 articles. 557 articles were excluded on the basis of their title or abstract, with 93 remaining for further evaluation. From these 93 articles, 9 where finally included in the meta-analysis [8-16]. Reasons of exclusion and numbers of 84 of these 93 studies are tabulated in Table 1. Five studies on 64-detector MDCTA and 4 studies on MDCTA with less than 64 detectors were included (32 detectors $\mathrm{n}=1,16$ detectors $\mathrm{n}=2,16$ and 4 detectors $\mathrm{n}=$ 1). One study was partially included (Table 2) since only a clearly defined fraction of the study patients were patients with negative biomarkers [15]. A flow diagram of the review process is given in Figure 1. In Additional file 2 the studies that were excluded were cited and classified according to Table 1.

Inter-observer agreement for the selection of articles between the two readers was $0.84,0.87$ and 1.0 for the first to third round respectively.(Cohen's Kappa).

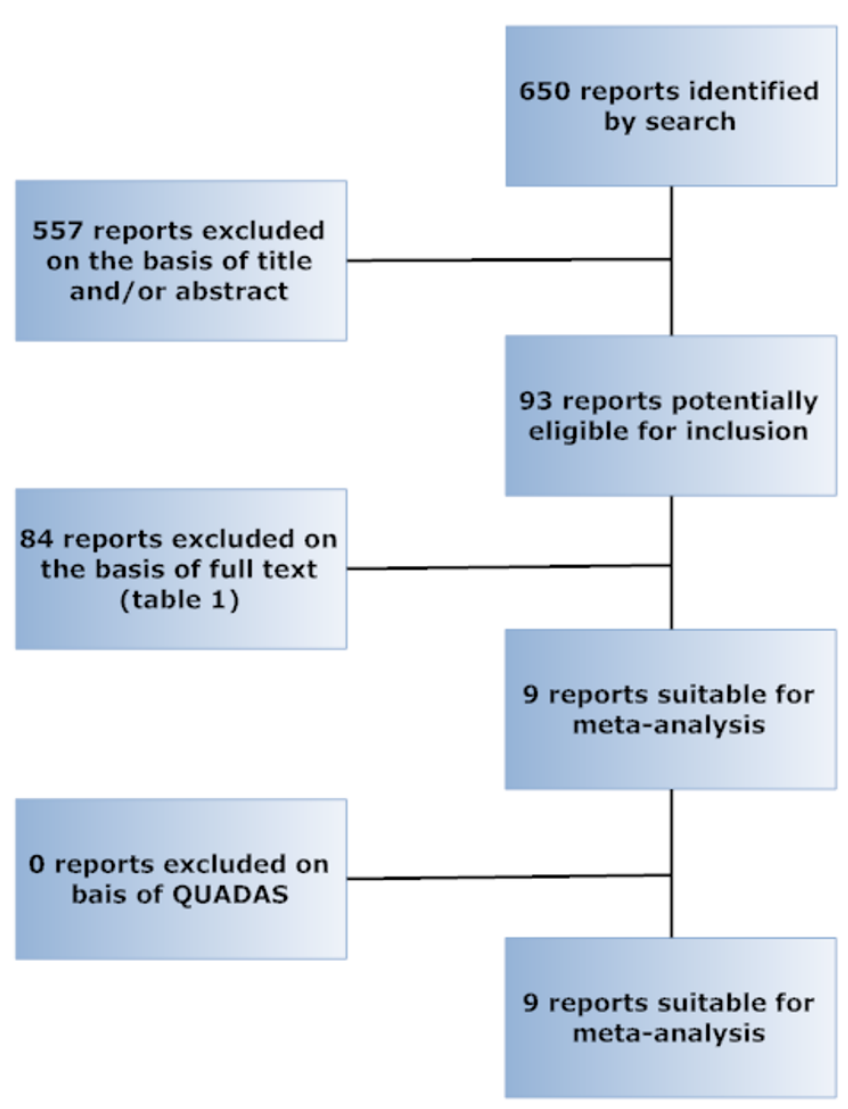

Figure 2

Flowdiagram of patient inclusion with patient categories used in summarizing data. In the studies analysed, from the eligible patients only a part was enrolled in the studies. The patients that were finally included was the subset of enrolled patients that completed the full protocol and that had diagnostic scans. Non assessable proportion (NAP) was the ratio of non-diagnostic patients or technical failures to the finally included patient.

Important study characteristics are displayed in Table 2.

All selected studies had a Quadas score of 14 .

\section{Data synthesis and statistical analysis}

A total of 566 patients where analysed. Only one study supplied information at the segment level, so that pooled analysis was only undertaken at the patient level.

Heterogeneity was not present among the studies when calculating the pooled DOR and NLR $\left(\mathrm{I}^{2}, 0 \%\right)$, but was considerable for SE, SP and PLR ( $\mathrm{I}^{2}, 43.0 \% ; 68.7 \% ; 62.2 \%$ respectively), which justifies our choice of a random effects model.

Publication bias was not present. (Intercept 0.89; $\mathrm{p}=$ 0.49); A funnel plot of the studies included is given in Fig- 


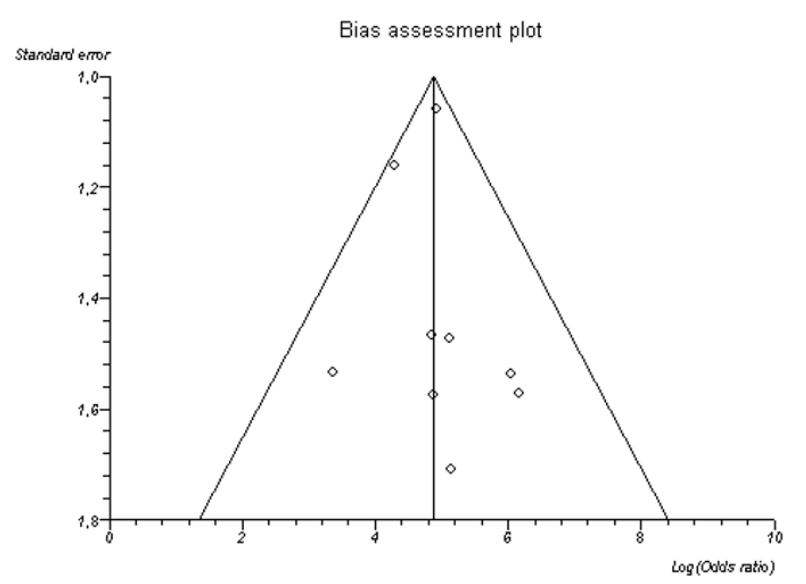

Figure 3

Graphical representation of publication bias. The dots, each representing one study are conforming to a triangular form, meaning that publication bias is low.

ure 3. It shows a good conformation to the ideal, funnel shaped distribution.

Raw data and pooled sensitivity, and specificity are summarized in Table 3.

The pooled sensitivity, specificity, NLR, PLR and the DOR are graphically displayed as forest plots in Fig. 4, 5, 6, 7, 8 and show the following values: Pooled DOR was 134.39 (95\%CI, 53.81-335.64). The pooled sensitivity and specificity were 0.95 (95\%CI, 0.90-0.98) and 0.90 (95\%CI, $0.87-0.93)$. The pooled NLR and PLR were 0.12 (95\%CI, $0.06-0.21)$ and 8.60 (95\%CI, 5.03-14.70).

SROC plot is found in Figure 9 and displays an excellent value for AUC (0.97, SE 0.01) and $Q^{*}$ (0.92, SE 0.01).
Pooled NAP was 0.03 (95\%CI, 0.01 to 0.07 )

The results of the logistic (meta-)regressions showed that none of the investigated variables had influence on the diagnostic performance or NAP.

\section{Discussion}

Our analysis of the available literature on MDCTA for the diagnosis of NSTEMI and/or UAP revealed that the overall pooled estimates of sensitivity and specificity on a patientlevel were good to excellent. Furthermore, the pooled estimates of NLR and PLR ( 0.12 and 8.60 repectively) and the results of the logistic (meta-) regressions revealed that the test can be used as a very reliable imaging modality to rule out NSTEMI and UAP in an acute setting, irrespective of scanner hardware and certain patient characteristics such as age or gender. The number of non-diagnostic scans was as low as 3\%, which showed that the technique seems to be robust. Moreover, detailed statistical analysis showed that this meta-analysis did not suffer from frequently occurring problems such as publication bias or extreme heterogeneity/inconsistency between studies.

A previous meta-analysis [34] that studied MDCTA in comparison with $\mathrm{CA}$ in a non-acute setting revealed important heterogeneity and some publication bias, especially when the analysis was done on a per segment basis, and demonstrated a prominent influence of the number of detectors on the number of non-assessable segments and diagnostic performance. Also the exclusion of segments before analysis had an important influence on diagnostic performance. The reason that these effects are statistically not demonstrable in the current study is probably due to the rather small sample and to the type of analysis, that was done exclusively on a per patient level.

This is an important observation: MDCTA can probably be used as a reliable technique to rule out NSTEMI or UAP

Table 3: Raw data from all the studies.

\begin{tabular}{|c|c|c|c|c|c|c|c|c|}
\hline \multirow{2}{*}{$\begin{array}{l}\text { Study } \\
\text { White } 8\end{array}$} & \multirow{2}{*}{$\begin{array}{c}\mathrm{TP} /(\mathrm{TP}+\mathrm{FN}) \\
10 / 12\end{array}$} & \multirow{2}{*}{$\begin{array}{c}\mathrm{FP} /(\mathrm{TN}+\mathrm{FP}) \\
55 / 57\end{array}$} & \multicolumn{3}{|c|}{ Sensitivity $(95 \% \mathrm{Cl})$} & \multicolumn{3}{|c|}{ Specificity $(95 \% \mathrm{Cl})$} \\
\hline & & & 0.83 & 0.52 & -0.98 & 0.96 & 0.88 & -1.00 \\
\hline Gallagher ${ }^{9}$ & $6 / 7$ & $72 / 78$ & 0.86 & 0.42 & -1.00 & 0.92 & 0.840 & -0.97 \\
\hline Hoffmann 10 & $5 / 5$ & $26 / 35$ & 1.00 & 0.48 & -1.00 & 0.74 & 0.57 & -0.87 \\
\hline Hoffmann"I & $14 / 14$ & $73 / 89$ & 1.00 & 0.77 & 1.00 & 0.82 & 0.73 & -0.89 \\
\hline Olivetti ${ }^{12}$ & $15 / 18$ & $13 / 13$ & 0.83 & 0.59 & -0.96 & 1.000 & 0.75 & -1.00 \\
\hline Sato ${ }^{13}$ & $21 / 22$ & $8 / 12$ & 0.95 & 0.77 & -1.00 & 0.89 & 0.52 & -0.98 \\
\hline Goldstein 14 & $8 / 8$ & $88 / 91$ & 1.000 & 0.63 & -1.00 & 0.97 & 0.91 & -0.94 \\
\hline Meijboom ${ }^{15}$ & $28 / 28$ & $4 / 5$ & 1.000 & 0.88 & -1.00 & 0.80 & 0.28 & -0.96 \\
\hline Rubinshtein 16 & $20 / 20$ & $35 / 38$ & 1.000 & 0.83 & -1.00 & 0.92 & 0.79 & -0.98 \\
\hline
\end{tabular}

TP: True positive.

TN: True negative.

FP: False positive.

FN: False negative.

95\% Cl: $95 \%$ Confidence intervals. 


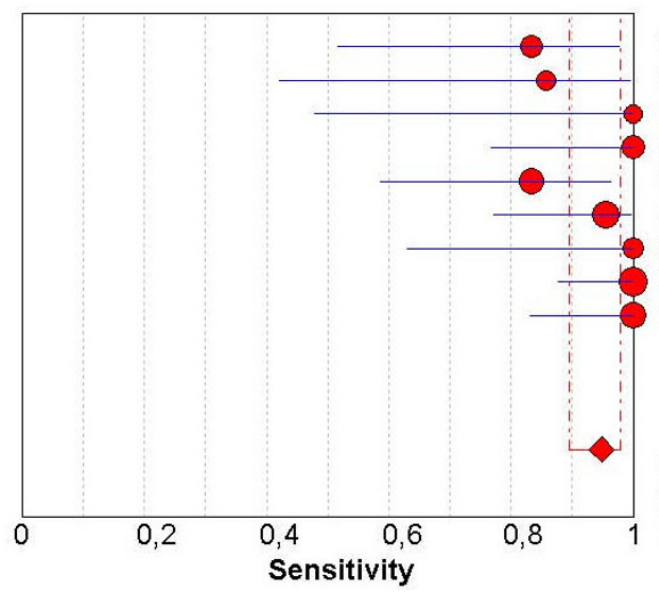

White 8

Ghallager 9

Hoffmann 10

Hoffmann 11

Olivetti 12

Sato 13

Goldstein 14

Meijboom 15

Rubinshtein 16

Pooled Sensitivity $=0,95(0,90$ to 0,98$)$

Chi-square $=14,04 ; \mathrm{df}=8(\mathrm{p}=0,0806)$

Inconsistency (I-square) $=43,0 \%$

Figure 4

Forest plot of sensitivity on a per patient basis.

in a acute setting and the test can be done very quickly and does not require complex patient preparation or logistic resources. The scan can be performed in the first hours after the onset of the clinical complaints and when negative, the patient can be discharged from the emergency department immediately. In the studies that were pooled in this meta-analysis, there was one randomized study that prooved that the average time from randomization to diagnosis was significantly shorter in the MDCTA arm than in the other arm (3.4 h versus $15 \mathrm{~h})[14]$.

Although the purpose of this study was not to formally study the reliability of MDCTA to exclude all causes of chest pain, it is apparent from the individual data from

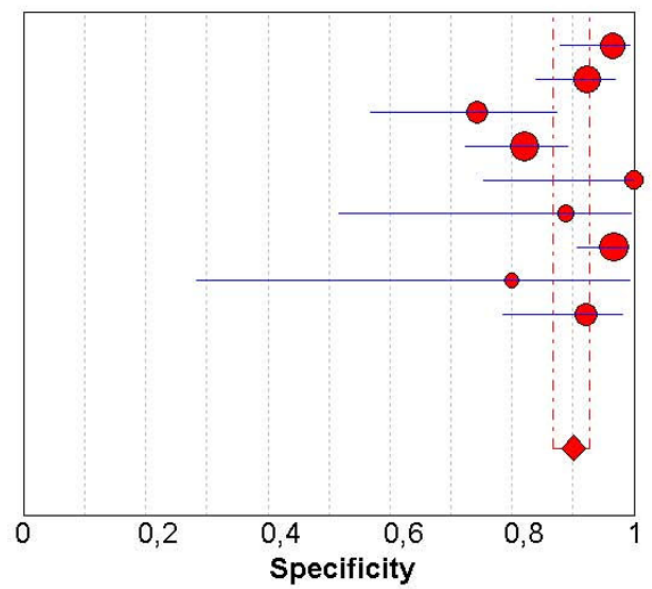

Sensitivity $(95 \% \mathrm{Cl})$

$0,83 \quad(0,52-0,98$

$0,86 \quad(0,42-1,00$

$1,00 \quad(0,48-1,00$

$1,00 \quad(0,77-1,00$

$0,83 \quad(0,59-0,96$

$0,95 \quad(0,77-1,00$

$1,00 \quad(0,63-1,00$

$1,00 \quad(0,88-1,00$

$1,00 \quad(0,83-1,00$
White 8

Ghallager 9

Hoffmann 10

Hoffmann 11

Olivetti 12

Sato 13

Goldstein 14

Meijboom 15

Rubinshtein 16

most studies in this series that alternative diagnoses

Other imaging techniques have been used to develop a quick and efficient triage strategy for acute chest pain in supporting its use in these circumstances [35]. In 2003 a the task force of the American College of Cardiology, the Heart Association and the American Society for perfusion imaging (MPI) [36]. The task fo gave a class 1 recommendation to the use of acute rest MPI (1)

Pooled Specificity $=0,90(0,87$ to 0,93$)$

Chi-square $=25,58 ; d f=8(p=0,0012)$

Inconsistency (I-square) $=68,7 \%$

\section{Figure 5}

Forest plot of specificity on a per patient basis. 


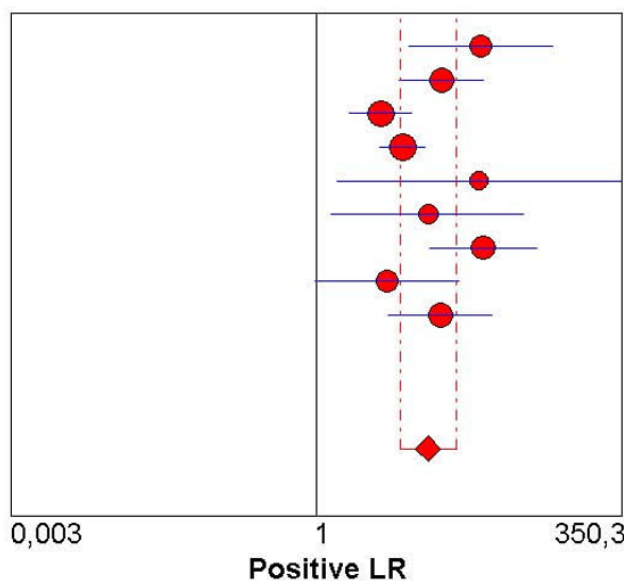

White 8

Ghallager 9

Hoffmann 10

Hoffmann 11

Olivetti 12

Sato 13

Goldstein 14

Meijboom 15

Rubinshtein 16
Positive LR (95\% Cl)

$23,75 \quad(5,95-94,85)$

$11,14 \quad(4,88-25,45)$

$3,47 \quad(1,91-6,31)$

$5,27 \quad(3,38-8,24)$

$22,84 \quad(1,49-350,32$

$8,59 \quad(1,35-54,64)$

$24,83 \quad(8,78-70,21)$

$3,93 \quad(0,98-15,73)$

$10,88 \quad(3,99-29,62)$

Random Effects Model

Pooled Positive LR $=8,60(5,03$ to 14,69$)$

Cochran- $Q=21,17 ; d f=8(p=0,0067)$

Inconsistency (I-square) $=62,2 \%$

Tau-squared $=0,3530$

Figure 6

Forest plot of positive likelihood ratio on a per patient basis. LR: Likelihood ratio.

acute coronary syndrome in whom initial markers and the ECG are non diagnostic. A randomized study [37] showed that MPI improves triage and that unnecessary hospitalisation was reduced among patients without acute ischaemia, without reducing appropriate admission for patients with acute ischemia. Wether MDCTA can be recommended as a substitute for MPI needs to be investigated further, since the total number of patients in this study is low.

Radiation dose may be a matter of debate. A clear disadvantage of MDCTA is the relatively high radiation dose that goes with the examination, with average doses ranging from 10 to $20 \mathrm{mS}$ [38]. How this relates to the dose of MPI now and in the future is a matter that goes beyond this discussion, but it may be anticipated that radiation dose reducing protocols for MDCTA will have an important impact [38].

Recently a few studies have described the use of three dimensional whole heart MRI for imaging the coronary tree in unstable patients. This technique has two advantages over MDCTA namely the lack of ionising radiation and no need to inject iodine containing contrast agents $[39,40]$.

Some limitations of this study have to be acknowledged. We put the presence of obstructive coronary disease and positive biomarkers on a similar level as an outcome of positive diagnosis. This is clearly an oversimplification

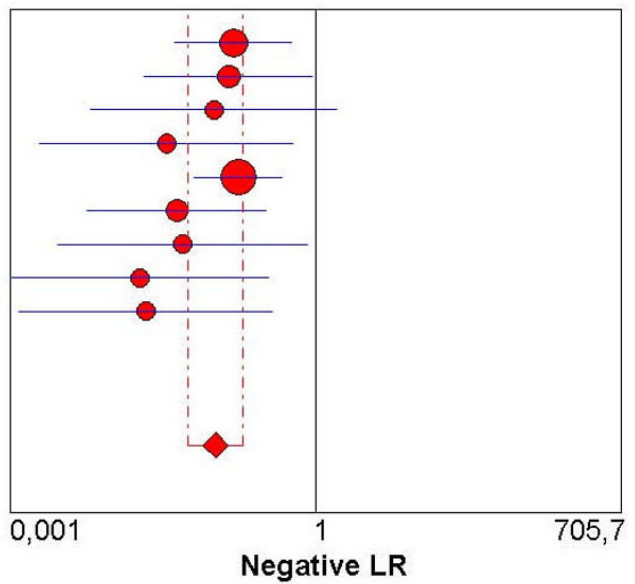

$\begin{array}{cc}\text { Negative LR }(95 \% \text { Cl } \\ 0,17 & (0,05-0,61) \\ 0,15 & (0,03-0,95) \\ 0,11 & (0,01-1,62) \\ 0,04 & (0,00-0,62 \\ 0,19 & (0,07-0,49) \\ 0,05 & (0,01-0,35 \\ 0,06 & (0,00-0,85) \\ 0,02 & (0,00-0,37) \\ 0,03 & (0,00-0,41)\end{array}$

Random Effects Model

Pooled Negative $L R=0,12(0,07$ to 0,21$)$

Cochran- $Q=6,24 ; d f=8(p=0,6204)$

Inconsistency (I-square) $=0,0 \%$

Tau-squared $=0,0000$

Figure 7

Forest plot of negative likelihood ratio on a per patient basis. LR: Likelihood ratio. 


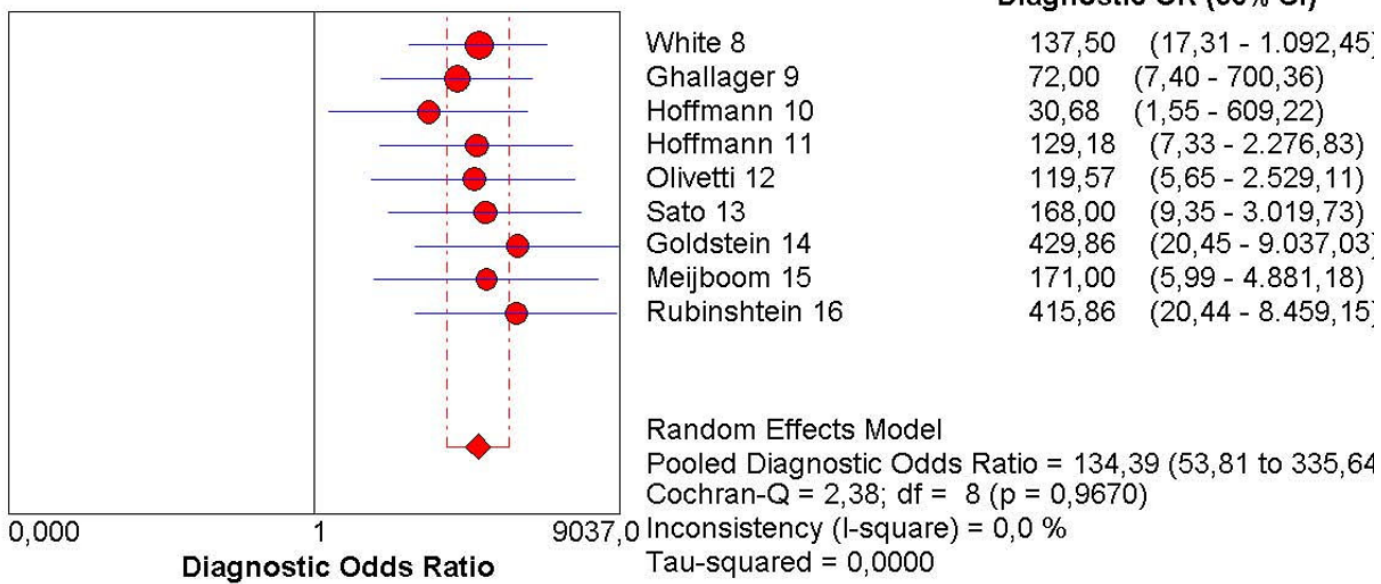

Figure 8

Forest plot of diagnostic odds ratio on a per patient basis. OR: Odds ratio.

and would suggest that every patient with obstructive disease has an ACS. This is obviously not the case. In the logistic meta-regression, there was however no influence of the type of outcome used on the diagnostic performance.

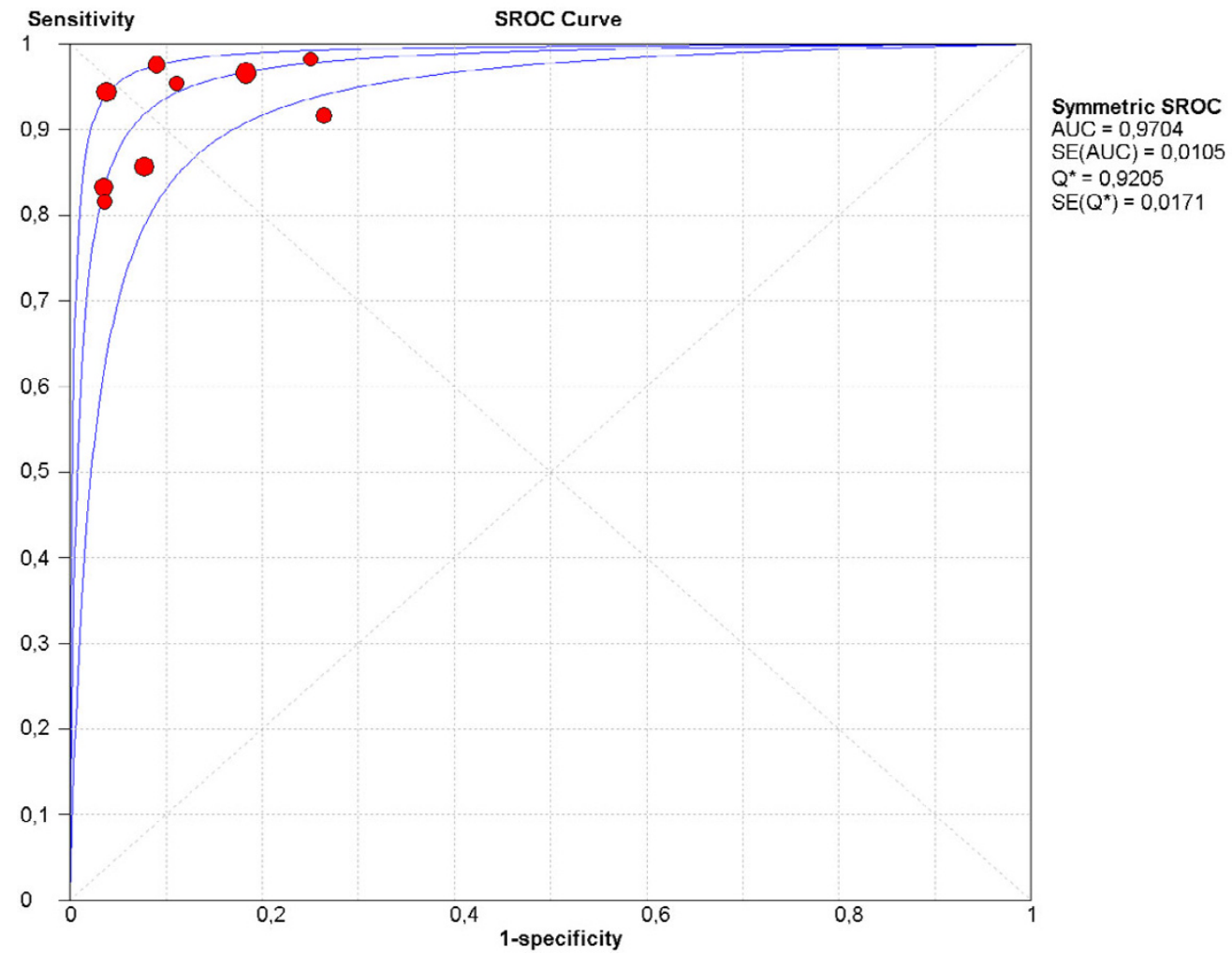

Figure 9

SROC curve of per patient analysis. AUC: Area under the curve. SE: Standard error. Q*: Point of intersection of the SROC curve where SE and SP are equal. 
The number of patients that was pooled in this analysis is rather low and the conclusions may be misleading to some degree. However, one of the studies incorporated was a randomized trial, and the conclusions drawn from this individual study were in line with the global conclusion, which is supporting the suggestion that the pooled conclusions are probably realistic [14]. Another, probably more important limitation is that detailed information on factors that technically limit MDCTA, such as high calcium score, irregular rythm, the presence of stents and dyspnea [41-44] was not mentioned in most studies, or at least not amenable to pooling. The same was true for pretest probability of the patient populations. This may cast some doubt on the potential to generalize the results to all patient populations.

The question may be asked why we did a meta-analysis on acute patients, since meta-analysis of MDCTA of the coronary arteries has already been performed previously [34]. We considered the studies in the acute setting as a different and interesting subgroup for two reasons. First, the studies that were included looked at outcome in a broader sense: clinical and/or conventional angiography. None of the studies included here has been included in this previous meta-analysis. Second the acute setting was supposed to include patients that may have a more dramatic clinical presentation in which MDCTA might result in more nondiagnostic scans. This selection resulted, as quoted already, in a small pooled group, with all attendant drawbacks.

\section{Conclusion}

The conclusion of this study is that MDCTA may be a safe and quick diagnostic technique to rule out NSTEMI or UAP in the emergency department. Future randomized studies should focus on including subtypes of patients that are known to be more difficult to image with MDCTA or to determine what the precise indication is for MDCTA and the place in the diagnostic algorithm. Cost-effectiveness studies should be done to investigate the economic impact of using this technique to evaluate chest pain in an acute setting.

\section{Competing interests}

The author(s) declare that they have no competing interests.

\section{Authors' contributions}

PV carried out the systematic search, statistical analysis, guarantor of study integrity, manuscript authoring and submission, data collection and prepared the manuscript. ID carried out the statistical analysis and contributed to the manuscript writing. OB, GS, CB and WW provided clinical advice and commented on the manuscript. All authors read and approved the final manuscript.

\section{Additional material}

\section{Additional file 1}

Search strategies. the data provided illustrate the search strategy followed Click here for file

[http://www.biomedcentral.com/content/supplementary/1471-

2261-7-39-S1.doc]

\section{Additional file 2}

Reasons for excluding articles. a list of excluded articles grouped in categories is given.

Click here for file

[http://www.biomedcentral.com/content/supplementary/14712261-7-39-S2.doc]

\section{References}

I. Lee TH, Rouan GW, Weisberg MC, Brand DA, Acampora D, Stasiulewicz C, Walshon J, Terranova G, Gottlieb L, Goldstein-Wayne B: Clinical characteristics and natural history of patients with acute myocardial infarction sent home from the emergency room. Am J Cardiol 1987, 60:219-224.

2. McCaig LF, Burt CW: National Hospital Ambulatory Medical Care Survey: 2002 emergency department summary. Adv Data 2004:I-34.

3. Pope JH, Aufderheide TP, Ruthazer R, Woolard RH, Feldman JA, Beshansky JR, Griffith JL, Selker HP: Missed diagnoses of acute cardiac ischemia in the emergency department. N Engl J Med 2000, 342: I 163-1 I70.

4. Hoffmann MH, Shi H, Schmitz BL, Schmid FT, Lieberknecht M, Schulze R, Ludwig B, Kroschel U, Jahnke N, Haerer W, Brambs HJ, Aschoff AJ: Noninvasive coronary angiography with multislice computed tomography. JAMA 2005, 293:247I-2478.

5. Leber AW, Knez A, von Ziegler F, Becker A, Nikolaou K, Paul S, Wintersperger B, Reiser M, Becker CR, Steinbeck G, Boekstegers P: Quantification of obstructive and nonobstructive coronary lesions by 64-slice computed tomography: a comparative study with quantitative coronary angiography and intravascular ultrasound. I Am Coll Cardiol 2005, 46: I 47-154.

6. Raff GL, Gallagher MJ, O'Neill WW, Goldstein JA: Diagnostic accuracy of noninvasive coronary angiography using 64-slice spiral computed tomography. I Am Coll Cardiol 2005, 46:552-557.

7. Hamon M, Biondi-Zoccai GG, Malagutti P, Agostoni P, Morello R, Valgimigli $M$, Hamon M: Diagnostic performance of multislice spiral computed tomography of coronary arteries as compared with conventional invasive coronary angiography: a metaanalysis. J Am Coll Cardiol 2006, 48: 1896-1910.

8. White CS, Kuo D, Kelemen M, Jain V, Musk A, Zaidi E, Read K, Sliker $C$, Prasad R: Chest pain evaluation in the emergency department: can MDCT provide a comprehensive evaluation? AJR Am J Roentgenol 2005, 1 85:533-540.

9. Gallagher MJ, Ross MA, Raff GL, Goldstein JA, O'Neill WW, O'neil B: The diagnostic accuracy of 64-slice computed tomography coronary angiography compared with stress nuclear imaging in emergency department low-risk chest pain patients. Ann Emerg Med 2007, 49:।25-136.

10. Hoffmann U, Pena AJ, Moselewski F, Ferencik M, Abbara S, Cury RC, Chae CU, Nagurney JT: MDCT in early triage of patients with acute chest pain. AJR Am J Roentgenol 2006, 187: 1240-1247.

II. Hoffmann U, Nagurney JT, Moselewski F, Pena A, Ferencik M, Chae CU, Cury RC, Butler J, Abbara S, Brown DF, Manini A, Nichols JH, Achenbach S, Brady TJ: Coronary multidetector computed tomography in the assessment of patients with acute chest pain. Circulation 2006, I | 4:225 I-2260.

12. Olivetti L, Mazza G, Volpi D, Costa F, Ferrari O, Pirelli S: Multislice $C T$ in emergency room management of patients with chest pain and medium-low probability of acute coronary syndrome. Radiol Med (Torino) 2006, I I I:I054-1063.

13. Sato $Y$, Matsumoto N, Ichikawa M, Kunimasa T, lida K, Yoda S, Takayama T, Uchiyama T, Saiti S, Nagao K, Tanaka H, Inoue F, Furu- 
hashi S, Takahashi M, Koyamam Y: Efficacy of multislice computed tomography for the detection of acute coronary syndrome in the emergency department. Circ J 2005, 69:|047-|05|

14. Goldstein JA, Gallagher MJ, O'Neill WW, Ross MA, O'Neil BJ, Raff GL: A randomized controlled trial of multi-slice coronary computed tomography for evaluation of acute chest pain. J Am Coll Cardiol 2007, 49:863-87I.

15. Rubinshtein R, Halon DA, Gaspar T, Jaffe R, Karkabi B, Flugelman MY, Kogan A, Shapira R, Peled N, Lewis BS: Usefulness of 64-slice cardiac computed tomographic angiography for diagnosing acute coronary syndromes and predicting clinical outcome in emergency department patients with chest pain of uncertain origin. Circulation 2007, I I 5:1762-1768.

16. Meijboom W, Mollet N, Van Mieghem C, Weustink A, Pugliese F, VanPelt N, Cademartirir F, Vourvouri F, de Jaegere P, Krestin GP, de Feyter PJ: 64-Slice computed tomography coronary angiography in patients with non-ST elevation acute coronary syndrome. Heart 2007, 93:1386-1392.

17. Deville W, Buntinx F, Bouter L, Montori V, de Vet H, van der Windt $D$, Bezemer P: Conducting systematic reviews of diagnostic studies: didactic guidelines. BMC Medical Research Methodology 2002, 2:9.

18. Staunton M: Evidence-based radiology: steps I and $\mathbf{2}$ - asking answerable questions and searching for evidence. Radiology 2007, 242:23-31.

19. Dodge JT Jr, Brown BG, Bolson EL, Dodge HT: Intrathoracic spatial location of specified coronary segments on the norma human heart. Applications in quantitative arteriography, assessment of regional risk and contraction, and anatomic display. Circulation 1988, 78: I |67- I I80.

20. Whiting P, Rutjes AW, Reitsma JB, Bossuyt PM, Kleijnen J: The development of QUADAS: a tool for the quality assessment of studies of diagnostic accuracy included in systematic reviews. BMC Med Res Methodol 2003, 3:25.

21. Bossuyt PM, Reitsma JB, Bruns DE, Gatsonis CA, Glasziou PP, Irwig LM, Lijmer JG, Moher D, Rennie D, de Vet HC: Towards complete and accurate reporting of studies of diagnostic accuracy: the STARD initiative. Fam Pract 2004, 21:4-10.

22. Thompson SG: Why sources of heterogeneity in meta-analysis should be investigated. BMJ 1994, 309:135I-I355.

23. Gavaghan DJ, Moore RA, McQuay HJ: An evaluation of homogeneity tests in meta-analyses in pain using simulations of individual patient data. Pain 2000, 85:415-424.

24. Thompson SG, Sharp SJ: Explaining heterogeneity in meta-analysis: a comparison of methods. Stat Med 1999, 18:2693-2708.

25. Higgins JP, Thompson SG: Quantifying heterogeneity in a metaanalysis. Stat Med 2002, 2 I:1539-1558.

26. Higgins JP, Thompson SG, Deeks JJ, Altman DG: Measuring inconsistency in meta-analyses. BMJ 2003, 327:557-560.

27. Egger M, Davey SG, Schneider M, Minder C: Bias in meta-analysis detected by a simple, graphical test. BMJ 1997, 3 I 5:629-634.

28. Sterne JA, Egger M, Smith GD: Systematic reviews in health care: Investigating and dealing with publication and other biases in meta-analysis. BMJ 200I, 323:I0I-105.

29. Berkey CS, Hoaglin DC, Mosteller F, Colditz GA: A random-effects regression model for meta-analysis. Stat Med 1995, I 4:395-4I I.

30. Littenberg B, Moses LE: Estimating diagnostic accuracy from multiple conflicting reports: a new meta-analytic method. Med Decis Making 1993, 13:313-321.

31. Moses LE, Shapiro D, Littenberg B: Combining independent studies of a diagnostic test into a summary ROC curve: data-analytic approaches and some additional considerations. Stat Med 1993, 12:1293-1316.

32. Irwig L, Tosteson AN, Gatsonis C, Lau J, Colditz G, Chalmers TC Mosteller F: Guidelines for meta-analyses evaluating diagnostic tests. Ann Intern Med 1994, I 20:667-676.

33. Walter SD: Properties of the summary receiver operating characteristic (SROC) curve for diagnostic test data. Stat Med 2002, 21:1237-1256.

34. Vanhoenacker PK, Heijenbrok-Kal MH, Vanheste R, Decramer I, Van Hoe L, Wijns W, Hunink MGM: Multidetector CT Angiography for assessment of coronary artery disease: Meta-analysis of Diagnostic Performance. Radiology 2007, 244:419-428.

35. loannidis JP, Salem D, Chew PW, Lau J: Accuracy of imaging technologies in the diagnosis of acute cardiac ischemia in the emergency department: a meta-analysis. Ann Emerg Med 200I, 37:47I-477.

36. Klocke FJ, Baird MG, Lorell BH, Bateman TM, Messer JV, Berman DS, O'Gara PT, Carabello BA, Russel RO, Verqueira MD, John Sutton MG, DeMaria AN, Udelson JE, Kennedy JW, Verani MS, Williams KA, Antman EM, Smith SC, Alper JS, Gregoratis G, Anderson JL, Hiratzka LF, Faxon DP, Hunt SA, Fuster V, Jacobs AK, Gibbons RJ, Russel RO: ACC/AHA/ASNC guidelines for the clinical use of cardiac radionuclide imaging - executive summary: a report of the American College of Cardiology/American Heart Association Task Force on Practice Guidelines (ACC/AHA/ASNC Committee to Revise the 1995 Guidelines for the Clinical Use of Cardiac Radionuclide Imaging). J Am Coll Cardiol 2003, 42:13|8-1333.

37. Udelson JE, Beshansky JR, Ballin DS, Feldman JA, Griffith JL, Handler J, Heller GV, Hendel RC, Pope JH, Ruthazer R, Spiegler EJ, Woolard $\mathrm{RH}$, Selker HP: Myocardial perfusion imaging for evaluation and triage of patients with suspected acute cardiac ischemia: a randomized controlled trial. JAMA 2002, 288:2693-2700.

38. Hausleiter J, Meyer T, Hadamitzky M, Huber E, Zankl M, Martinoff S, Kastrati A, Schomig A: Radiation dose estimates from cardiac multislice computed tomography in daily practice: impact of different scanning protocols on effective dose estimates. Circulation 2006, I I3:1305-1310.

39. Sato $\mathrm{Y}$, Matsumoto N, Yoda S: Whole-heart coronary magnetic resonance angiography in a patient with unstable angina. Int J Cardiol 2006, I I I:309-3 I0.

40. Sato Y, Komatsu S, Matsumoto N, Tani S, Kunimasa T, Masubuchi M, Kunimoto S, Kasmaki Y, Takahashi M, Uchiyama T, Saito S: Whole heart coronary magnetic resonance angiography for the detection of coronary artery stenosis and atherosclerotic coronary artery plaque in a patient with unstable angina. Int J Cardiol 2007, I I 5:262-264.

4I. Cademartiri F, Runza G, Mollet NR, Luccichenti G, Belgrano M, Bartolotta TV, Galia M, Midiri M, Pozzi MR, Krestin GP: Impact of intravascular enhancement, heart rate, and calcium score on diagnostic accuracy in multislice computed tomography coronary angiography. Radiol Med (Torino) 2005, I I 0:42-5I.

42. Cademartiri F, Mollet NR, Runza G, Belgrano M, Malagutti P, Meijboom BW, Midiri M, de Feyter PJ, Krestin GP: Diagnostic accuracy of multislice computed tomography coronary angiography is improved at low heart rates. Int J Cardiovasc Imaging 2006, 22: $101-105$

43. Cademartiri F, Mollet NR, Lemos PA, Saia F, Runza G, Midiri M, Krestin GP, de Feyter PJ: Impact of coronary calcium score on diagnostic accuracy for the detection of significant coronary stenosis with multislice computed tomography angiography. Am J Cardiol 2005, 95: I 225- I 227.

44. Sirineni GK, Kalra MK, Pottala K, Waldrop S, Syed M, Tigges S: Effect of contrast concentration, tube potential and reconstruction kernels on MDCT evaluation of coronary stents: an in vitro study. Int J Cardiovasc Imaging 2007, 23:253-263.

\section{Pre-publication history}

The pre-publication history for this paper can be accessed here:

http://www.biomedcentral.com/1471-2261/7/39/prepub 